Freie Vorträge

\title{
Die Stellung der Chemotherapie im Rahmen der kombinierten Krebsbehandlung
}

\author{
I. Mogos, C. Neacsu, M. Andreescu \\ und E. Herbay-Bukarest/Rumänien (a.G.)
}

Um die therapeutischen Ergebnisse beim Brustkrebs im allgemeinen, und diejenigen der fortgeschrittenen Stadien des Brustkrebses im besonderen zu verbessern, führen die Autoren eine kombinierte Untersuchung durch (klinisch und mit markierten Cytostatica), um festzustellen :

1. Die klinischen Ergebnisse und die Nebenwirkungen, die bei Patienten mit Brustkrebs beobachtet wurden, die mit verschiedenen Cytostatica (Endoxan, Clafen, Sarcolysine, Thio-Tepa, IOB-82, 5 FU usw.) und unterschiedlichen Verabreichungsarten (allgemein und besonders örtlich-regional, intra-arteriell) mit unterschiedlichen Dosierungen und Geschwindigkeiten behandelt wurden.

2. Überlebensraten nach jedem der oben erwähnten Verabreichungsverfahren.

3. Therapeutische Wirksamkeit von cytostatischen Kombinationen (Polychemotherapie) und/oder mit Hormon-Immunstrahlentherapie und Chirurgie.

Zwei weitere Maßstäbe der therapeutischen Wirksamkeit - Thermographie und Chromosomenabweichungen - wurden ebenfalls diskutiert, die die Einführung einer rationellen und kontrollierten Chemotherapie in die Klinik ermöglichen.

\section{Cytogenetische Aspekte bei Tumoren der Brustdrüse}

\author{
M. Andreescu, M. Mateescu, J. Mogos \\ C. Neacsu und E. Herbay-Bukarest/Rumänien (a.G.)
}

Die Autoren sind der Ansicht, daß sowohl dem biologischen Vorgang der Neoplasie wie den Zellveränderungen nach nichtchirurgischer Behandlung beim Menschen auch cytogenetische, quantitative und qualitative Reorganisationen zugrunde liegen. Ziel dieser Arbeit ist es, den Nachweis für diese Aspekte zu führen. In diesem Zusammenhang wurde das plötzliche Auftreten möglicher cytogenetischer Modifikationen im Tumor unterstrichen, das im Vergleich zum anfänglichen Bild (vor Behandlung) unter nichtchirurgischer Behandlung zu verzeichnen war. 